\title{
NONNEGATIVELY CURVED CONTACT MANIFOLDS
}

\section{S. I. GOLDBERG ${ }^{1}$}

\begin{abstract}
A compact simply connected contact Riemannian manifold with positive sectional curvature is homeomorphic with a sphere if its contact structure is normal and regular. In dimension 3 the regularity condition is omitted, and a stronger theorem is proved.
\end{abstract}

1. Introduction. The sphere theorem says that a compact simply connected $\delta$-pinched Riemannian manifold with $\delta>1 / 4$ is homeomorphic with a sphere, and in the odd-dimensional case, if $\delta=1 / 4$, the same conclusion prevails. Since all complex and quaternionic projective spaces and the Cayley plane are 1/4-pinched, this is the best result for even-dimensional manifolds. It is not known, however, whether the theorem can be improved for odd-dimensional manifolds. On the other hand, Siu and Yau [10] showed that a compact Kaehler manifold with $\delta>0$ is analytically homeomorphic with complex projective space $C P_{n}$. In this paper, the proper odd-dimensional analogue of the Siu-Yau theorem is obtained. It is an improvement of the sphere theorem for this class of manifolds and answers a question posed by Sasaki [9]. In dimension 3 an even stronger theorem is obtained by invoking a theorem of Hamilton [6]. The results obtained lend credibility to the classical Poincaré conjecture. Finally, to our knowledge, the first examples of nonregular contact structures on compact simply connected three-manifolds are given.

2. Contact manifolds. An almost contact structure $\left(\phi, X_{0}, \omega\right)$ on a $(2 n+1)$ dimensional $C^{\infty}$ manifold $M$ is given by a linear transformation field $\phi$, a vector field $X_{0}$ called the characteristic field, and a 1-form $\omega$ with

$$
\omega\left(X_{0}\right)=1, \quad \phi X_{0}=0 \text { and } \phi^{2}=-1+\omega(\cdot) X_{0},
$$

where $I$ is the identity transformation (see $[2]$ for definitions and other properties). If $M$ has such a structure, it is called an almost contact manifold. In this case, a Riemann metric $g$ can be found such that

$$
\omega=g\left(X_{0}, \cdot\right), \quad g(\phi X, Y)=-g(X, \phi Y)
$$

for any vector fields $X$ and $Y$. Such a metric is called an associated metric and is clearly not unique. The resulting structure is then called an almost contact metric or Riemannian structure.

A $(2 n+1)$-dimensional $C^{\infty}$ manifold is called a contact manifold if it carries a global 1-form $\omega$ with the property that $\omega \wedge(d \omega)^{n} \neq 0$ everywhere. The classical

Received by the editors November 12, 1984. Presented at the Colloquium on Differential Geometry sponsored by the University of Debrecen and the Hungarian Academy of Sciences on August 28, 1984.

1980 Mathematics Subject Classification. Primary 53C15; Secondary 53C20.

Key words and phrases. Contact Riemannian manifolds, curvature, three-manifolds.

${ }^{1}$ Research supported by the Natural Sciences and Engineering Research Council of Canada. 
example is the bundle of unit tangent vectors to an oriented $(n+1)$-dimensional manifold. An odd-dimensional sphere $S^{2 n+1}$ possesses a contact structure which is not of this type. In fact, every compact simply connected homogeneous contact manifold is a circle bundle over a homogeneous Hodge manifold [3]. Conversely, a compact Hodge manifold $B$ has a contact manifold canonically associated with it as a circle bundle with $B$ as base space.

A contact manifold $M$ with contact form $\omega$ has an underlying almost contact Riemannian structure $\left(\phi, X_{0}, \omega, g\right)$ such that $g(X, \phi Y)=d \omega(X, Y)$. If the characteristic field $X_{0}$ is a Killing vector field with respect to $g$, then

$$
\begin{gathered}
2\left(\nabla_{X} \omega\right)(Y)=d \omega(X, Y), \\
g\left(R\left(X, X_{0}\right) Y, X_{0}\right)=g(X, Y)-\omega(X) \omega(Y)=g(\phi X, \phi Y),
\end{gathered}
$$

and

$$
S\left(X, X_{0}\right)=2 n \omega(X),
$$

where $\nabla_{X}$ denotes covariant differentiation with respect to $g$ in the 'direction' $X, R$ is the Riemannian curvature tensor, and $S$ is the Ricci tensor. Thus, the sectional curvature of a plane section containing $X_{0}$ is positive, and the Ricci curvature in the direction $X_{0}$ is $2 n$.

If the almost compact structure $J$ on $M \times R$ defined by

$$
J(X, f d / d t)=\left(\phi X-f X_{0}, \omega(X) d / d t\right)
$$

is integrable, the almost contact structure $\left(\phi, X_{0}, \omega\right)$ is said to be normal, and $M$ is called a normal contact manifold. In this case, $X_{0}$ is a Killing vector field with respect to the associated metric $g$. A normal contact Riemannian manifold may be characterized by

$$
[X, Y]+\phi[\phi X, Y]+\phi[X, \phi Y]-[\phi X, \phi Y]=\{X \omega(Y)-Y \omega(X)\} X_{0} .
$$

A distribution $V$ is associated with $\omega$ as follows. Let

$$
V_{p}=\left\{X \in T_{p} M \mid d \omega(X, Y)=0 \forall Y \in T_{p} M\right\} .
$$

Then $\operatorname{dim} V_{p}=1$. Thus, $V$ is an integrable distribution and determines a 1dimensional foliation of $M$. The contact structure is said to be regular if this foliation is regular, that is, if for each point $p \in M$ there are Frobenius coordinates around $p$ such that different slices belong to different leaves.

3. Curvature and the Poincaré conjecture. In this section we obtain the 'odd-dimensional Siu-Yau theorem'. But first, we recall a more rigid result [5]. A compact simply connected normal regular contact manifold of positive sectional curvature and constant scalar curvature is globally isometric to a sphere (with metric not necessarily the constant curvature metric).

The regularity condition was subsequently removed by E. Moskal in his thesis (unpublished). This is the proper odd-dimensional analogue of the following improvement of a theorem of M. Berger: A compact Kaehler manifold of positive sectional curvature and constant scalar curvature is isometric to $C P_{n}$ with the constant holomorphic curvature metric.

In the Siu-Yau theorem, the condition on the scalar curvature is dropped. If the condition on the scalar curvature is omitted in Moskal's statement, the following theorem is obtained. 
THEOREM 1. Let $M$ be a compact simply connected contact Riemannian manifold of dimension $2 n+1$ with positive sectional curvature. If the contact structure is normal and regular, then $M$ is homeomorphic with $S^{2 n+1}, n>1$.

A considerably stronger theorem for $n=1$ will be obtained below.

ProOF. The base manifold $B$ of the Boothby-Wang fibration of $M$ carries a Kaehler metric of positive curvature (see [5]). $B$ is therefore homeomorphic with complex projective space $C P_{n}$. Moreover, the fibration has nonzero Euler class. Thus, from the homotopy sequences of the fiberings $S^{1} \rightarrow S^{2 n+1} \rightarrow C P_{n}$ and $S^{1} \rightarrow M \rightarrow B, \pi_{i}(M) \cong \pi_{i}\left(S^{2 n+1}\right), i>1$. Since $M$ is simply connected, it is therefore of the same homotopy type as $S^{2 n+1}$. Hence, by Smale's solution of the generalized Poincare conjecture [11], we conclude that $M$ is homeomorphic with $S^{2 n+1}$ for $n>1$.

Martinet [7] showed that a compact and orientable 3-manifold $M$ possesses a contact structure. If it is normal, and $S+\lambda g$ is positive definite for some $\lambda<2$, where $g$ is an associated metric, then $g$ may be deformed to a metric with positive Ricci curvature. To see this, consider the deformation defined by $\tilde{g}=a g+b \omega \otimes \omega$, where $a$ and $b$ are constants with $a>0$ and $a+b>0$. The inverse matrix $\left(\tilde{g}^{i j}\right)$ of $\left(\tilde{g}_{i j}\right)$ is given by

$$
\tilde{g}^{i j}=\frac{1}{a} g^{i j}-\frac{b}{a(a+b)} X_{0}^{i} X_{0}^{j} .
$$

Let $\Gamma_{j k}^{i}$ denote the coefficients of the Riemannian connection of $g$, namely, the Christoffel symbols, and consider the tensor field $W_{j k}^{i}=\tilde{\Gamma}_{j k}^{i}-\Gamma_{j k}^{i}$. Then

$$
W_{j k}^{i}=-\frac{b}{a}\left(\phi_{\cdot j}^{i} \omega_{k}+\phi_{\cdot k}^{i} \omega_{j}\right)+\frac{b}{2(a+b)} X_{0}^{i}\left(\nabla_{j} \omega_{k}+\nabla_{k} \omega_{j}\right)
$$

Since $X_{0}$ is a Killing vector field, the Lie derivative of $g$ with respect to $X_{0}$ vanishes, so that

Substituting this in

$$
W_{j k}^{i}=-\frac{b}{a}\left(\phi_{\cdot j}^{i} \omega_{k}+\phi_{\cdot k}^{i} \omega_{j}\right)
$$

$$
\tilde{R}_{\cdot j k l}^{i}=R_{\cdot j k l}^{i}+\nabla_{l} W_{j k}^{i}-\nabla_{k} W_{j l}^{i}+W_{r l}^{i} W_{j k}^{r}-W_{r k}^{i} W_{k l}^{r},
$$

where the $R_{\cdot j k l}^{i}$ and $\tilde{R}_{\cdot j k l}^{i}$ are the components of the curvature tensors of the metrics $g$ and $\tilde{g}$, respectively,

$$
\begin{aligned}
\tilde{R}_{\cdot j k l}^{i}= & R_{\cdot j k l}^{i}+\frac{b}{a}\left(2 \phi_{\cdot j}^{i} \phi_{k l}+\phi_{\cdot k}^{i} \phi_{j l}-\phi_{\cdot l}^{i} \phi_{j k}\right. \\
& \left.+\omega_{l} \nabla_{k} \phi_{\cdot j}^{i}+\omega_{j} \nabla_{k} \phi_{\cdot l}^{i}-\omega_{k} \nabla_{l} \phi_{\cdot j}^{i}-\omega_{j} \nabla_{l} \phi_{\cdot k}^{i}\right) \\
& +\frac{b^{2}}{a^{2}} \omega_{j}\left(\omega_{k} \delta_{l}^{i}-\omega_{l} \delta_{k}^{i}\right) .
\end{aligned}
$$

Thus, since $M$ is normal,

$$
\left(\nabla_{X} \phi\right) Y=g(X, Y) X_{0}-\omega(Y) X
$$

from which

$$
\tilde{S}=S-\frac{2 b}{a} g+\frac{2 b}{a^{2}}[(2 n+1) a+n b] \omega \otimes \omega .
$$


Putting $n=1$ gives

$$
\tilde{S}=S-\frac{2 b}{a} g+\frac{2 b}{a^{2}}(3 a+b) \omega \otimes \omega .
$$

If $X$ is vertical, that is, if $X=t X_{0}$,

$$
\tilde{R}_{i j} X^{i} X^{j}=t^{2}\left[R_{i j} X_{0}^{i} X_{0}^{j}+\frac{2 b}{a^{2}}(2 a+b)\right]=2 t^{2}\left[1+\frac{b}{a^{2}}(2 a+b)\right]
$$

since $S\left(X_{0}, \cdot\right)=2 \omega$. If $X$ is horizontal, that is, if it belongs to the distribution defined by $\omega=0$, then

$$
\tilde{R}_{i j} X^{i} X^{j}=R_{i j} X^{i} X^{j}-\frac{2 b}{a} g_{i j} X^{i} X^{j}
$$

By choosing $b=a^{2}-a$, we see that $\tilde{R}_{i j} X^{i} X^{j}>0$ if $X$ is vertical. Moreover, setting $a=(2-\lambda) / 2$, where $\lambda<2$, then for any horizontal $X$,

$$
\tilde{R}_{i j} X^{i} X^{j}=R_{i j} X^{i} X^{j}+\lambda g_{i j} X^{i} X^{j}
$$

We conclude that $\tilde{S}$ is positive definite if $S+\lambda g, \lambda<2$, is positive definite.

The above proof is valid for $n>1$ as well, that is, the metric $g$ of any $(2 n+1)$ dimensional normal contact Riemannian manifold can be deformed to a metric of positive Ricci curvature if $S+\lambda g, \lambda<2(1-\sqrt{1-1 / n})$, is positive definite. It is shown in [12] that if $S+2 g$ is positive definite on $\left(M^{3}, g\right)$, then it is a rational homology sphere. The following is a much stronger statement.

THEOREM 2. A compact simply connected three-dimensional normal contact Riemannian manifold $(M, g)$ with $S+\lambda g$ positive definite for some $\lambda<2$ is homeomorphic with $S^{3}$.

PROOF. We have just shown that if $S+\lambda g$ is positive definite for some $\lambda<2$, then the metric $g$ may be deformed to a metric $\tilde{g}$ with positive Ricci curvature. Therefore, by a theorem of Hamilton [6], $\tilde{g}$ can be deformed to a metric of constant positive sectional curvature, and so since $M$ is simply connected, it is homeomorphic with $S^{3}$.

COROLLARY. A compact simply connected three-dimensional normal contact Riemannian manifold with nonnegative sectional curvature is homeomorphic with $S^{3}$.

4. Remarks. (a) A compact three-dimensional regular contact manifold $M$ is normal since the base manifold of the Boothby-Wang fibration of $M$ is a Riemann surface. Moreover, if $M$ is also simply connected, it is homeomorphic with $S^{3}$. This is a consequence of the classification of circle bundles on surfaces. More general results in this direction are (i) a compact simply connected Seifert fibre space (admitting an $S^{1}$-action, not necessarily free) is homeomorphic with $S^{3}$. This result is due to Raymond [8]. (ii) Every $C^{r}$ foliation of a compact orientable three-manifold $N$ by circles is $C^{r}$ diffeomorphic to a Seifert fibration. This is due to Epstein [4]. Hence, if $N$ is simply connected, it is homeomorphic with $S^{3}$. It generalizes Theorem 1 of $[3]$ when $n=1$.

(b) If the Poincaré conjecture is true, the proof of Theorem 1 can be extended to the three-dimensional case. 
(c) To our knowledge, no examples of nonregular contact structures on compact simply connected three-dimensional manifolds have appeared in the literature. The examples given by Abe and Erbacher [1] of compact three-manifolds with a nonregular contact structure are not simply connected. We owe the following to $\mathrm{R}$. Kulkarni.

Let

$$
S^{3}=\left\{\left.(z, w) \in C^{2}|| z\right|^{2}+|w|^{2}=1\right\}, \quad z=x+i y, w=u+i v .
$$

For $\alpha \in R, \alpha \neq 0$, consider the $R$-action $\phi_{\alpha}: R \times S^{3} \rightarrow S^{3}$, where $\phi_{\alpha}(\theta,(z, w))=$ $\left(e^{i \theta} z, e^{i \alpha \theta} w\right)$. Then

$$
X=\left.\frac{d}{d \theta} \phi_{\alpha}(\theta,(z, w))\right|_{\theta=0}=i(z, \alpha w)=(y, x,-\alpha v, \alpha u)
$$

is a vector field tangential to the orbits. Note that since $\alpha \neq 0$, the action has no fixed points. Let

$$
\omega=(x d y-y d x)+(1 / \alpha)(u d v-v d u) .
$$

It is easily seen that

(a) $\omega \wedge d \omega \neq 0$ everywhere on $S^{3}$;

(b) $\left\langle\left. X\right|_{S^{3}}\right.$ is the null space of $\left.d \omega\right|_{S^{3}}$;

(c) $\left.\omega(X)\right|_{S^{3}}=1$.

If $\alpha=1$ or -1 , one gets the Hopf fibration. Let $\alpha=p / q$, where $p, q$ are relatively prime. Then the $R$-action is not effective. It factors through the action of $R / 2 \pi q Z$, and so it induces a foliation of $S^{3}$ by circles. However, if $\alpha \neq 1$ or -1 , this foliation is not a principal fibration.

If $\alpha \notin Q$, the $R$-action is effective. The orbits of $(1,0)$ and $(0,1)$ are circles; the rest are arcs. Thus, for $\alpha \neq 1$ or -1 the contact structure is nonregular.

On any manifold, the set of nonregular contact structures forms an open dense subset in the set of all contact structures. Openness is clear. As for denseness, one need only vary a given regular contact form a little so that the flow of the associated line field is not periodic. This may be achieved by a local construction.

(d) In the corollary it is not difficult to show that the sectional curvatures can be taken to be greater than $-3 / 2$ everywhere.

\section{REFERENCES}

1. K. Abe and J. Erbacher, Nonregular contact structures on Brieskorn manifolds, Bull. Amer. Math. Soc. (N.S.) 81 (1975), 407-409.

2. D. E. Blair, Contact manifolds in Riemannian geometry, Lecture Notes in Math., Vol. 509, Springer-Verlag, Berlin and New York, 1976.

3. W. M. Boothby and H. C. Wang, On contact manifolds, Ann. of Math. (2) 68 (1958), 721-734.

4. D. B. A. Epstein, Periodic flows on three-manifolds, Ann. of Math. (2) 95 (1972), 66-82.

5. S. I. Goldberg, Rigidity of positively curved contact manifolds, J. London Math. Soc. $\mathbf{4 2}$ (1967), 257-263.

6. R. S. Hamilton, Three-manifolds with positive Ricci curvature, J. Differential Geom. 17 (1982), 255-306.

7. J. Martinet, Formes de contact sur les variétés de dimension 3, Proc. Liverpool Singularities Sympos. II, Springer-Verlag, 1971, pp. 142-163.

8. F. Raymond, Classification of the actions of the circle on 3-manifolds, Trans. Amer. Math. Soc. 131 (1968), 51-78. 
9. S. Sasaki, Almost contact manifolds, Part 3, Lecture Notes, Math. Inst., Tôhoku Univ., Japan, 1968.

10. Y.-T. Siu and S.-T. Yau, Compact Kahler manifolds of positive bisectional curvature, Invent. Math. 59 (1980), 189-204.

11. S. Smale, The generalized Poincaré conjecture in higher dimensions, Bull. Amer. Math. Soc. (N.S.) 66 (1960), 373-375.

12. S. Tanno, The topology of contact Riemannian manifolds, Illinois J. Math. 12 (1968), 700-717.

DEPARTMENT OF MATHEMATICS, UNIVERSity OF ILlinOIS, 1409 W. GREEN STREET, URBANA, ILLINOIS 61801 AND

DePARTMENT OF MATHEMATICS AND STATISTICS, QUeEN'S UNIVERSity, KingSTON, ONTARIO, CANADA 